\title{
Correction to: Genetic analysis and gene mapping of the orange flower trait in Chinese cabbage (Brassica rapa L.)
}

\author{
Ning Zhang • Huamin Zhang • Yanjing Ren • \\ Lin Chen • Jie Zhang • Lugang Zhang
}

Published online: 15 July 2019

(C) Springer Nature B.V. 2019

\section{Correction to: Mol Breeding (2019) 39: 76 \\ https://doi.org/10.1007/s11032-019-0984-5}

The original version of this article unfortunately contains incorrect supplementary materials.

The corrected supplementary materials can be found online.

Publisher's note Springer Nature remains neutral with regard to jurisdictional claims in published maps and institutional affiliations.

The online version of the original article can be found at https://doi.org/10.1007/s11032-019-0984-5

Electronic supplementary material The online version of this article (https://doi.org/10.1007/s11032-019-1019-y) contains supplementary material, which is available to authorized users.

N. Zhang $\cdot$ H. Zhang $\cdot$ Y. Ren $\cdot$ L. Chen $\cdot$ J. Zhang $\cdot$

L. Zhang $(\bowtie)$

State Key Laboratory of Crop Stress Biology for Arid Areas,

College of Horticulture, Northwest A\&F University,

Yangling 712100 Shaanxi, China

e-mail: lugangzh@163.com

Present Address:

H. Zhang

Henan Chinese Chives Engineering Technology Research Center, Pingdingshan Academy of Agricultural Sciences,

Pingdingshan 467001 Henan, China 